\title{
Generation and saturation of large scale flows in flute turbulence
}

\author{
I. Sandberg ${ }^{1,2} *$, H. Isliker ${ }^{2}$, V. N.Pavlenko ${ }^{3}$, K. Hizanidis ${ }^{1}$ and L. Vlahos ${ }^{2}$ \\ ${ }^{1}$ Department of Electrical and Computer Engineering, \\ National Technical University of Athens, GR-157 73 Athens, Greece \\ ${ }^{2}$ Department of Physics, Aristotle University of Thessaloniki, GR-54124 Thessaloniki, Greece \\ ${ }^{3}$ Department of Astronomy and Space Physics, Uppsala University, \\ Box 515, SE-751 20 Uppsala, Sweden \\ ASSOCIATION EURATOM-HELLENIC REPUBLIC
}

(Received October 14, 2004)

PACS numbers: 52.35.Py, 52.35.Mw, 52.35.Ra

\begin{abstract}
The excitation and suppression of large-scale anisotropic modes during the temporal evolution of a magnetic-curvature-driven electrostatic flute instability is numerically investigated. The formation of streamer-like structures is attributed to the linear development of the instability while the subsequent excitation of the zonal modes is the result of the non-linear coupling between linearly grown flute modes. When the amplitudes of the zonal modes become of the same order as that of the streamer modes, the flute instabilities get suppressed and poloidal (zonal) flows dominate. In the saturated state that follows, the dominant large-scale modes of the potential and the density are self-organized in different ways, depending on the value of the ion temperature.
\end{abstract}

* E-mail address: sandberg@central.ntua.gr 


\section{INTRODUCTION}

Electrostatic turbulence, driven by spatial gradients, is believed to be the dominant source of anomalous transport in magnetically confined fusion plasma. Special emphasis has been given lately on the properties of large scale anisotropic flows generated by the drifttype turbulence, due to the critical role they play in the regulation of the low-frequency drift instabilities and consequently of the levels of turbulent transport [1], [2]. The spontaneous generation of large-scale flows driven by electrostatic wave turbulence has been experimentally observed in plasma discharges in various machines, e.g. in TEXT, RFX, DIII-D [3], [4]. Zonal flows (ZFs) correspond to potential structures which spatially depend on the radial coordinate $x$ (the coordinate along the axis of plasma inhomogeneity), while radial flows or streamers (STs) are radially elongated potential structures which spatially depend on the poloidal coordinate $y[5]$. In tokamak plasmas, ZFs have the ability to limit the radial size of turbulent eddies through the shear decorrelation mechanism [6], and hence to regulate turbulent transport. Hence, the high plasma confinement modes are attributed to the presence of large scale poloidal flows (zonal flows). Streamers, on the other hand, are ineffective at inhibiting radial transport and, due to their long radial correlation length, may lead to enhanced or bursty levels of transport [7].

In general, the formation of such flows in plasmas is ensured when the small-scale dynamics permit the cascade of energy towards large scales. In this case, the mechanism for flow formation is commonly attributed to the Reynolds stress forces generated by the small-scale fluctuations. It was shown [10] that the flow formation occurs when the underlying smallscale turbulence supports waves propagating in the direction of the plasma inhomogeneity and when gradients in the turbulent Reynolds stress exist. Similar is the mechanism responsible for the formation of the zonal structures observed in rotating planetary atmospheres as on the Earth [8] or on Jupiter [9]. During the last years, several theoretical models have been proposed to describe the properties of strongly anisotropic large scale flows in relation to drift [10]- [13], ion temperature gradient (ITG) [14], electron temperature gradient (ETG) 
[15], [16], and magnetic-curvature-driven flute (interchange) instabilities [17].

The aim of the present work is the numerical investigation of the generation and saturation of large scale anisotropic flows by an evolving magnetic-curvature-driven flute instability. Flute modes are low-frequency $\left(\omega \ll \omega_{c i}\right)$ electrostatic oscillations of a non-uniform magnetoplasma which are elongated along the magnetic field $k_{\|}=0$ (flute limit) and become unstable due to the combined effects of the density inhomogeneity and the curvature of the magnetic field lines. In the flute limit, plasma particles do not follow the Boltzmann relation and cannot cancel the charge separation induced by the difference between the perturbed electron and ion curvature drift velocities. This leads to the development of an electric field component perpendicular to the magnetic field direction that amplifies the initial perturbation, which becomes unstable. The flute instability is also termed interchange instability, as it tends to interchange "flux tubes" of different pressure causing convective transport. Thus, it is considered to be one of the most dangerous instabilities in thermonuclear fusion devices.

In the cold ion limit $\left(T_{i}=0\right)$, the generation and saturation of zonal flows due to the development of the flute instability has been numerically investigated by Das et al. [18]. The authors showed, among others, that for large values of the dynamical viscosity and diffusion coefficients the non-linear effects attributed to the short scale fluctuations become rather weak and the generation of the zonal flows is inhibited. For finite ion temperature, the generation of large scale flows in flute turbulence has been studied analytically in Ref. [17] on the basis of a scale separation between the short scale turbulent fluctuating fields and the large scale flows. As it was shown, streamers can be generated through both linear and non-linear mechanisms, while zonal flows can be excited only non-linearly. Furthermore, the role of the ion temperature can be of great importance due to the ion diamagnetic drift non-linearity which appears in the Reynolds stress tensor for finite ion Larmor radius.

In order to extend the theory of the formation of large scale flows in flute turbulence, we here numerically investigate the non-linear dynamics of the flute instability using the more general model of the flute equations as presented in Ref. [19], for the case of electron-proton 
plasmas. This model is a generalization of [17] and [18], as it includes both electron and ion curvature drifts and it incorporates the effects associated to finite ion pressure fluctuations. Finite ion temperature has manifold influence on the development and evolution of the flute instability; the ion curvature drift leads to an increase of the growth rates, while the ion diamagnetic drift stabilizes the short scale flute instabilities, leading to a narrowing of the spectrum of the linearly excited modes. Furthermore, the diamagnetic component of the polarization drift non-linearity, attributed to the finite ion Larmor radius, is expected to lead to a direct cascading of the fluctuation energy towards short scales, according to Ref. [21], suppressing in this way the generation of the large scale flows. Thus, the conditions for the nonlinear generation of large-scale structures in the frame of our model are more complex. In order to elucidate these conditions, we perform numerical studies of the nonlinear evolution of the flute instability. We will focus on the description of the excitation, interaction and suppression of the largest-scale anisotropic modes, i.e. the zonal and the streamer modes. Zonal modes are defined here as the modes with $k_{y}=0$ and with a small but finite radial scale lengths $k_{x}^{-1}$. Streamer modes are defined as the modes with $k_{x}=0$ and small but finite poloidal wavenumbers $k_{y}$.

In the following section, we briefly present the model of equations that describes the evolution of the flute instability, and in section III we present the numerical results. Finally, in Sec. IV follow the summary and the conclusions.

\section{BASIC EQUATIONS}

A weakly inhomogeneous magnetized plasma with characteristic inhomogeneity scale length $L_{n}$ along the radial axis $x$ is considered. The magnitude $B(x)$ and the unit vector $\mathbf{b}$ of the curved magnetic field are modeled by $B(x)=B_{0}(1-x / R)$ and $\mathbf{b}=\hat{z}-(z / R) \hat{x}$, respectively, where $R\left(>L_{n}\right)$ is the curvature radius of the magnetic field lines. Starting from the two-fluid plasma equations [22], and assuming flute-type $\left(k_{\|}=0\right)$, quasi-neutral, electrostatic oscillations, it is found that the magnetic-curvature-driven flute modes are de- 
scribed by the following set of dimensionless coupled equations for the perturbed electrostatic potential $\phi$ and density $n[19]$ :

$$
\begin{gathered}
\left(\partial_{t}-\tau v_{n} \partial_{y}\right) \nabla_{\perp}^{2} \phi+v_{g}(1+\tau) \partial_{y} n=\tau \operatorname{div}\left\{\nabla_{\perp} \phi, n\right\}+\left\{\nabla_{\perp}^{2} \phi, \phi\right\}+\mu \nabla^{4}(\phi+\tau n) \\
\left(\partial_{t}+v_{g} \partial_{y}\right) n+\left(v_{n}-v_{g}\right) \partial_{y} \phi=\{n, \phi\}+D \nabla^{2} n,
\end{gathered}
$$

where $\{f, g\}=\hat{z} \times \nabla f \cdot \nabla g$ denotes the Poisson bracket. The system of Eqs. $(1,2)$ describes the non-linear interaction of the flute modes and admits the excitation of large scale flows. It generalizes other models $[17,18]$, including rigorously the magnetic curvature drifts of both electrons and ions, and the finite ion Larmor radius effects. The first equation originates from the quasi-neutrality condition $\nabla_{\perp} \cdot\left[n\left(\overrightarrow{v_{i}}-\overrightarrow{v_{e}}\right)\right]=0$, and the second one results from the electron continuity equation. For the derivation of these equations, the perpendicular perturbed velocities of the electron and the ion fluids were analytically expressed in terms of a sum of their associated perturbed drift velocities induced by low-frequency electrostatic perturbations. For the ion fluid velocity, a standard successive approximation was used, based on the drift velocity ordering, according to which the ion polarization and the ion stress tensor drift velocities are much smaller compared to the electric and the diamagnetic drifts (see, for example Ref. [19]). In Eqs. (1,2), the electrostatic potential has been normalized by $T_{e} / e$, the time by the ion cyclotron frequency $\omega_{c i}$, the lengths by the ion Larmor radius $\rho=c_{s} / \omega_{c i}$ defined at the electron temperature (here $c_{s}^{2}=T_{e} / m_{i}$ ), the density by the unperturbed plasma density $n_{0}$, and the temperatures by the electron temperature $T_{e}$. The ion temperature is now denoted by $\tau\left(=T_{i} / T_{e}\right)$. In dimensional units, the electron curvature and diamagnetic drift velocities are given by $v_{g}=2 c_{s}^{2} /\left(R \omega_{c i}\right)$ and $v_{n}=c_{s}^{2} /\left(L_{n} \omega_{c i}\right)$, while the viscous and diffusion coefficients $\mu$ and $D$ are given by $\mu=(3 / 10)\left(T_{i} \nu_{i} / \omega_{c i} m_{i}\right), D=$ $m_{e} T_{e} \nu_{e} /(e B)^{2}$, where $\nu_{j}$ denotes the collision frequency of the plasma particles $(j=i, e)$.

The inclusion of finite ion temperature effects leads to the appearance of two additional linear terms on the left hand side of Eq. (1). The first term is due to the ion diamagnetic drift, while the second one is due to the ion curvature drift. Assuming small amplitude 
perturbations and applying the usual Fourier expansion for the perturbed amplitudes, we linearize Eqs. $(1,2)$ and determine the frequency $\omega_{k}=\frac{1}{2}\left[k_{y}\left(v_{g}-\tau v_{n}\right)\right]$, and the growth rate

$$
\gamma_{k}=\frac{1}{2}\left[\sqrt{\frac{4 k_{y}^{2}}{k_{\perp}^{2}} v_{g}\left(v_{n}-v_{g}\right)(1+\tau)-k_{y}^{2}\left(\tau v_{n}+v_{g}\right)^{2}+k_{\perp}^{4}(D-\mu)^{2}}-k_{\perp}^{2}(D+\mu)\right] .
$$

of the unstable flute modes.

The presence of finite ion temperature leads to a decrease of the frequency and subsequently of the characteristic wave velocities of the flute modes. For $\tau<v_{g} / v_{n}$ the flute modes propagate in the same direction with the electron curvature drift velocity, while for $\tau>v_{g} / v_{n}$ it propagates in the opposite direction (i.e. in that of the ion diamagnetic drift velocity). Furthermore, for finite ion temperature $\tau$, the growth rate of the most unstable flute modes increases due to the ion curvature drift, while the spectrum of the unstable flute modes gets narrower due to the stabilization of the short wavelength modes by the ion diamagnetic drift.

In the temporal evolution of the flute instability, the inverse energy cascade may lead to the generation of large scale flows [20]. However, since the most unstable flute modes are those of zero radial wave number $\left(k_{x}=0\right)$ and small but finite poloidal wave number $\left(k_{y} \neq 0\right)$, i.e. the streamer modes, it is expected that streamer-pattern large scale flows can be formed through the linear development of the flute instability. On the contrary, it is evident from Eq. (3) that linear excitation of zonal modes is not possible since $\gamma_{k}<0$ for $k_{y}=0$. As one may see from Eqs. $(1,2)$, there exist several non-linear terms which determine the cascading properties of the flute turbulence. The polarization drift nonlinearity $\left\{\nabla_{\perp}^{2} \phi, \phi\right\}$ is responsible for the energy cascading towards large scale flows [20], while the convective non-linearity $\{n, \phi\}$ is known to cascade energy towards short scales [18]. Moreover, the diamagnetic component of the polarization drift non-linearity $\tau \operatorname{div}\left\{\nabla_{\perp} \phi, n\right\}$, which is attributed to the finite ion Larmor radius, is expected to lead to direct cascading of the fluctuation energy towards short scales [21]. Hence, the description of the formation of large scale flows during the temporal evolution of flute turbulence is more complicated with respect to the electrostatic drift wave turbulence. 


\section{NUMERICAL RESULTS}

We have studied numerically the temporal evolution of the system described by Eqs. $(1,2)$ and the subsequent excitation of large scale flows by using a dealized pseudospectral code in a numerical grid of $128 \times 128$ points. The marching in time is performed with a fourth order Runge-Kutta technique with adaptive step-size. We have imposed periodic boundary conditions and considered a physical domain in the $x y$ plane of area $\Delta x \times \Delta y=$ $[(-30 \pi, 30 \pi) \times(-30 \pi, 30 \pi)]$. The minimum finite wavenumber which can be resolved with

our scheme is $k_{0}=k_{x 0}=k_{y 0}=0.033$. In the numerical simulations, we have chosen the normalized (over the sound velocity) values of the electron diamagnetic and the curvature drift velocities to be $v_{n}=0.03$ and $v_{g}=0.01$, respectively, while the viscosity and the diffusion coefficients are fixed at $D=\mu=0.1$. The selection of these values allows the linear growth of a sufficient range of short scale fluctuations [18], enabling the subsequent excitation of zonal modes through the inverse energy cascade mechanism. The initial conditions for the potential and the density perturbations consist of an isotropic spectrum of small amplitude and randomly phased Fourier modes of the form

$$
\begin{aligned}
& \phi(x, y)=\sum_{k_{x}} \sum_{k_{y}} \hat{\phi} \exp \left(i \vec{k} \cdot \vec{r}+i \alpha_{k_{x}, k_{y}}\right) \\
& n(x, y)=\sum_{k_{x}} \sum_{k_{y}} \hat{n} \exp \left(i \vec{k} \cdot \vec{r}+i \beta_{k_{x}, k_{y}}\right)
\end{aligned}
$$

where $\hat{\phi}=0.001, \hat{n}=0.0005$, and $\alpha_{k_{x}, k_{y}}, \beta_{k_{x}, k_{y}}$ are randomly generated initial phases.

\section{A. Non-linear dynamics of flute turbulence}

The temporal evolution and suppression of the flute instabilities lead to the development of saturated anisotropic spectra with clear indication of the formation of large scale flows. As one may see in Fig. 1, the structures of these flows depends crucially on the value of the ion temperature. In the presentation of the numerical results which follows, we focus on the description of the three major distinct phases associated with the evolution of the dominant large-scale flute modes. 
a. Excitation of Streamer Modes The first phase of the evolution of the flute instabilities is characterized by the growth of the linearly unstable flute modes. During this phase, patterns of radial streamers of the fluctuating potential and density are formed in the real space, since for given finite poloidal wave number $k_{y}$ the flute modes of maximum growth rate are those of $k_{x}=0$, i.e. the streamer modes. In Fig. 2a, the evolution of the three largest streamer modes for the potential are depicted. The linear profiles during the initial evolution (for $0<t \lesssim 400$ ) correspond to the linear growth of the streamer modes while the numerical values of the growth rates are in good agreement with that predicted by the linear theory (c.f. Eq. (3)). The growth of the streamer modes continues until a suppression mechanism sets on. As we can see from Fig. 2a, the duration of the growth is different for each streamer mode as the onset of the suppression depends on the mode's wavenumber. In general, the smaller the streamer mode is, the faster, and consequently at smaller amplitudes, it gets suppressed.

We should note here that during the very initial phase of the temporal evolution, a short time interval appears with properties much different than the linear ones which follow. This is attributed to the numerical response of the system to the arbitrary initial conditions we imposed. Hence, the initial abrupt growth of the streamer modes (see Fig. 2a, for $0<t \leq 20$ ) indicates an inverse energy cascade from the initial short scale fluctuations towards larger scales. Also, the decrease in the power of the total potential fluctuations $\sum_{k_{\perp}} k_{\perp}^{2}\left|\Phi_{k}\right|^{2}$ (see Fig. 7 , for $0<t \leq 180$ ) can be attributed to the linear damping of the shorter scale modes according to Eq.(3).

b. Excitation of Zonal Modes When the amplitude of the flute perturbations reaches a critical value, zonal modes are excited. This is a purely non-linear effect and arises due to the non-linear coupling of the linearly grown flute modes. In Fig. 2b, the excitation and the evolution of the three largest zonal modes is depicted. These modes are generated almost simultaneously (around $t=180$ ) and grow exponentially with similar growth rates. The growth rate of the most unstable zonal mode (the one with $\vec{k}=\left(k_{0}, 0\right)$ ) has approximately double the value of that of the dominant linear instability (that with $\vec{k}=\left(0, k_{0}\right)$ ). This is 
due to the quadratic nature of the nonlinear terms in Eqs. $(1,2)$ and indicates that the zonal modes grow under the action of at least a couple of linearly amplified flute modes of small but finite poloidal wave number.

c. Suppression of anisotropic modes $\&$ Saturation When the potential amplitude of the dominant non-linearly growing zonal mode becomes of order similar to the potential amplitude of the most grown streamer mode, both amplitudes start to oscillate in an outof phase manner fashion. This can be clearly seen in Fig. 3 and indicates the coupling between the dominant and largest anisotropic modes of the potential. The result of this mode coupling is the suppression of the streamer instability and the saturation of the growth of the zonal mode. Similar description accounts also for the evolution and suppression of the smaller zonal and streamer modes (cf. Fig. 2), since the mechanism is qualitatively the same. The linear flute-instabilities, as the streamer modes, are suppressed through the shear stabilization mechanism $k_{x}^{2} \Phi$, which is provided by the growing zonal modes. This leads to the subsequent saturation of the zonal modes as well, since the flute modes which were responsible for the zonal growth got suppressed by the zonal modes themselves. In the saturated state which follows the suppression of the flute instabilities, the amplitudes of the zonal and the dominant streamer modes of the potential remain almost constant. On the contrary, the smaller streamer modes get damped (cf. Fig. 2).

In order to shed some light onto the coupling mechanism between the dominant anisotropic modes, we have investigated the role of the largest and most grown isotropic modes. These are the modes with wavenumbers $\vec{k}=\left( \pm k_{0}, \pm k_{0}\right)$, which provide the necessary matching conditions for a three-wave coupling between the largest anisotropic modes. We have carried out a numerical simulation by setting the amplitudes of these large scale isotropic modes equal to zero. The numerical results regarding the temporal evolution of the dominant anisotropic potential modes for this particular case are presented in Fig. 4. As one may see, the excitation and the initial growth of the zonal mode do not seem to be significantly dependent on the presence of the large scale isotropic modes. However, the growth of the zonal mode terminates at a smaller amplitude compared to that of the 
dominant streamer mode. This occurs because the smaller scale instabilities that support the growth of the dominant zonal mode got suppressed by the smaller zonal modes. As a direct consequence, the most unstable flute instability does not get suppressed. These results show that the large-scale isotropic modes, being the coupling carrier between the largest anisotropic modes, support the mechanism: a) for the further growth of the zonal mode, b) for the suppression of the largest streamer mode and hence, c) for the subsequent formation of the poloidal flow. From the above, it is evident that short scale fluctuations can be significant for the generation of zonal flows, and large scale isotropic modes for the suppression of the flute instabilities. Hence, one may claim that a wide range of modes contributes to the growth of the zonal modes, which enable the subsequent suppression of the flute instabilities. Consequently, models based on scale separation between the short scale fluctuations and large scale flows seem not to be suitable for the complete description of the evolution of flute instabilities.

In Fig. 5 we present the temporal evolution of the largest anisotropic and isotropic modes. In the saturated state, which follows the suppression of the instabilities, the amplitudes obey an oscillatory behavior as they remain coupled and exchange energy with each other. However, as one may note there is an important difference between the dominant saturated modes of the potential and the density. Concerning the potential fluctuations, the largest zonal mode is always the dominant, while the amplitudes of the isotropic modes are much smaller compared to the anisotropic ones. On the contrary, the amplitude of the largest zonal mode of the density can be of the same order or smaller compared to that of the isotropic large scale modes. Hence, it becomes evident that the modes of the potential and the density are organized differently on reaching the dynamical equilibrium that determines the saturated state of the flute turbulence. This result is not surprising since the plasma density response is not Boltzmannian in the flute limit, and hence the density fluctuations are expected to behave differently than the potential ones.

d. Finite Ion Temperature Effects Performing numerical calculations for different values of the ion temperature $\tau$ and keeping the same values for the rest of the parameters, we have 
investigated the influence of ion temperature on the evolution and saturation of the flute instability. Qualitatively, the description is similar to that presented in the previous section. This is particularly true concerning the excitation of the dominant anisotropic modes (cf. Fig. 6). In that phase, the ion temperature modifies the growth rates of the instabilities, as expected, due to the presence of the ion curvature drift which co-drives together with the electron curvature drift the excitation of the linear flute modes. Consequently, the amplitudes of the saturated modes depend also on the value of the ion temperature. In Fig. 7, we have plotted the evolution of the power of the potential fluctuation $\sum_{k_{\perp}} k_{\perp}^{2}\left|\Phi_{k}\right|^{2}$ for different values of the ion temperature. In the lower panel, we note that as $\tau$ increases the saturation level of the power of the potential fluctuations also increases. On the contrary, in the upper panel, it is clearly shown that further increase of the ion temperature, above $\tau>2$, leads to a decrease of the saturated level. A similar description accounts also for the amplitudes of the saturated large scale modes. In addition to this, the relative amplitudes between the saturated modes are also changing for different values of the ion temperature. As one may see in Fig. 6, the saturated potential spectra e.g. for $\tau=1$ are dominated by the zonal mode while for $\tau=3$ the saturated amplitudes of streamers and zonal modes become of similar order. This modifies the structures of the saturated potential in the real space as shown in Fig. 1. The saturated amplitudes of the density modes seem to be even more sensitive to the value of the ion temperature $\tau$. As one may see in the right-hand-side panels of Fig. 6, the dominant modes of density are of zonal type for $\tau=0$ and $\tau=1$, but not when the ion temperature adopts the values $\tau=0.5$ and $\tau=3$. An additional effect associated with the increase of the ion temperature is the appearance of secondary excitations and subsequent saturations of streamer modes. This can be seen for instance in the lower panel of Fig. 6 and in the upper panel of Fig. 7. Hence, it seems that the dynamical equilibrium between the coupled modes in the saturation state is sensitive to ion temperature effects.

In order to determine, the role of the diamagnetic component of the polarization drift non-linearity, $\tau \operatorname{div}\left\{\nabla_{\perp} \phi, n\right\}$, which appears when finite ion Larmor effects are considered, 
we have performed a set of numerical simulations with and without this term. As major result, we found that its presence always leads to smaller saturated amplitudes for the large scale modes and similarly for the total power of the potential fluctuations $\sum_{k} k_{\perp}^{2}\left|\Phi_{k}\right|^{2}$. Furthermore, we notice that the absence of $\tau \operatorname{div}\left\{\nabla_{\perp} \phi, n\right\}$ increases the number of secondary excitations of the streamer modes. These results justify the prediction of Ref. [21], according to which the diamagnetic component of the polarization drift non-linearity leads to the direct cascade towards short scale modes. Subsequently, it is expected that the ion temperature through this non-linear mechanism: a) suppresses the inverse cascade towards large scale modes, and b) stabilizes the secondary excitations of streamers. From the above, it follows that the increase of the amplitudes of the saturated modes for $0<\tau<3$ and the appearance of the secondary instabilities are related to the linear terms proportional to $\tau$ in Eq.(1), and hence to the increase of the linear growth rate.

\section{DISCUSSION-CONCLUSIONS}

We have numerically investigated the excitation and suppression of large scale anisotropic modes as a result of the development of the flute instability. The initial formation of the streamer flow is attributed to the linear growth of the streamer modes, while the subsequent formation of the zonal flow is the result of the excitation of large-scale zonal modes through the inverse energy cascading mechanism. The most grown instabilities are the largest-scale ones and saturate last. The numerical results show that their suppression can be attributed to the non-linear interaction between the largest scale flute modes. The saturated state which follows is characterized by the domination of the largest zonal mode for the potential. However, the complexity increases when ion temperature effects are considered, especially concerning the type of the dominant saturated mode of the density. In general, as long the value of ion temperature is small, the amplitudes of the saturated modes increase as the ion temperature increases. On the contrary, for large values of the ion temperature, the saturated amplitudes decrease due to the suppression of the inverse energy cascade, which 
can be attributed to the diamagnetic component of the polarization drift non-linearity. It was also shown that modes of various scales are rather significant for the suppression of the flute instabilities. Hence, theoretical models based on the scale separation approximation, or zero models which incorporate only short scale fluctuations, STs and ZFs, may only be adequate to describe just the excitation of the zonal modes. The complex behavior concerning the suppression and saturation of the instabilities can possibly be described in terms of mode competition type models with three competitive large-scale populations, namely the streamer, the zonal and the largest scale isotropic modes for each fluctuating quantity, i.e. for the potential and the density.

\section{ACKNOWLEDGMENT}

This work was supported by the European Fusion Programme (EURATOM) and the Greek General Secretariat of Research and Technology. The sponsors do not bear any responsibility for the contents in this work. 


\section{REFERENCES}

[1] W. Horton, Rev. Mod. Phys. 71, 735 (1999).

[2] P. W. Terry, Rev. Mod. Phys. 72, 109 (2000).

[3] S. Coda, M. Porkilab, and K. H. Burrell, Phys. Rev. Lett. 86, 4835 (2001).

[4] P. A. Politzer, Phys. Rev. Lett.84, 1192 (2000).

[5] F. H. Busse, Chaos 4, 123 (1994).

[6] H. Biglari, P. H. Diamond, and P. W. Terry, Phys. Fluids B 2, 1 (1990).

[7] P. Beyer, S. Benkadda, X. Garbet, and P. H. Diamond, Phys. Rev. Lett. 85, 4592 (2001).

[8] O. G. Onishchenko, O. A. Pokhotelov, R. Z. Sagdeev, P. K. Shukla, and L. Stenflo, Nonlinear Processes in Geophysics, 11 (2004).

[9] P. K. Shukla, P. K. Dwivedi and L. Stenflo, New J. Phys. 5 (2003).

[10] P. H. Diamond and Y. B. Kim, Phys. Fluids B3, 1626 (1991).

[11] M. A. Malkov, P. H. Diamond and M. N. Rosenbluth, Phys. Plasmas 8, 5073 (2001).

[12] M. A. Malkov and P. H. Diamond, Phys. Plasmas 8, 3996 (2001).

[13] P. N. Guzdar, R. G. Kleva, N. Chakrabati, Phys. Plasmas 11, 3324 (2004).

[14] B. N. Rogers, W. Dorland, and M. Kotschenreuther, Phys. Rev. Let 85, 5336 (2000).

[15] J. Li and Y. Kishimoto, Phys. Plasmas 9, 1241 (2002).

[16] P. K. Shukla, L. Stenflo, J. Plasma Phys. 70, 41 (2004).

[17] Z. Andrushchenko and V. P. Pavlenko, Phys. Plasmas 9, 11 (2002).

[18] A. Das, A. Sen, S. Mahajan, and P. Kaw, Phys. Plasmas 8, 5104 (2001).

[19] I. Sandberg and P. K. Shukla, Phys. Plasmas 11, 542 (2004). 
[20] A, Hasegawa, C, G. Maclennan, and Y. Kodama, Phys. Fluids 22, 2122 (1979).

[21] Y. Kodama and V. P. Pavlenko, Phys. Rev. Lett. 60, 1506 (1988).

[22] S. I. Braginskii, in Reviews of Plasma Physics, Vol.1, Edited by M. A. Leontovich, (Consultants Bureau, New York, 1965) 


\section{List of figure captions}

FIG. 1 Iso-contour lines of the potential in the saturation state for (a) $\tau=0$, (b) $\tau=1$, and (c) $\tau=3$. In Subfigs. (a) and (b) the zonal modes largely dominate over the streamer modes. Hence, patterns of zonal flows are formed. For $\tau=3$, the amplitude of the dominant streamer mode is of the same order as the one of the zonal mode, resulting in the pattern of Subfig. (c).

FIG. 2 Evolution and saturation of the largest streamer $\left|\Phi\left(0, m k_{0}\right)\right|^{2}$ (upper panel) and zonal modes $\left|\Phi\left(m k_{0}, 0\right)\right|^{2}$ (lower panel) of the potential. Here, $m=1$ (solid line), $m=2$ (dashed line) and $m=3$ (dotted line) for $\tau=1$, where $k_{0}$ is the minimum wavenumber of the system. The shorter zonal and streamer mode instabilities saturate earlier and at lower amplitudes compared to the largest ones. In the saturated state, the amplitudes of the zonal modes and the largest streamer mode remain constant, while the smaller streamer modes get damped.

FIG. 3 Oscillating behavior of the dominant anisotropic modes for the potential during the suppression of the instabilities, for $\tau=0$. The zonal mode $\left|\Phi\left(k_{0}, 0\right)\right|^{2}$ is depicted with the solid line, and the streamer mode $\left|\Phi\left(0, k_{0}\right)\right|^{2}$ with the dashed line.

FIG. 4 Evolution of the dominant zonal (solid line) and streamer (dashed line) modes in the absence of the dominant isotropic modes $\vec{k}\left( \pm k_{0}, \pm k_{0}\right)$, for $\tau=1$. The growth of the zonal mode stops at low amplitude, and as a result the dominant flute instability (streamer mode) does not get suppressed.

FIG. 5 Temporal evolution and saturation of the dominant isotropic and anisotropic modes for (a) the potential and (b) the density in the cold ion limit. The zonal mode, $\vec{k}\left(k_{0}, 0\right)$ is depicted with solid line, the streamer mode $\vec{k}\left(0, k_{0}\right)$ with dashed line and the isotropic

modes $\vec{k}\left(k_{0}, k_{0}\right), \vec{k}\left(k_{0},-k_{0}\right)$ with the smaller dashed and the dotted lines, respectively. The amplitudes of the isotropic modes in the saturation state are significant for the density fluctuations.

FIG. 6 Evolution of the dominant modes of the potential and the density for different values of the ion temperature: (a) $\tau=0.5$ (b) $\tau=1$ and (c) $\tau=3$. The zonal mode is 
depicted with solid lines, the streamer mode with dashed lines, and the isotropic modes $\vec{k}\left(k_{0}, k_{0}\right) \vec{k}\left(k_{0},-k_{0}\right)$ with smaller dashed and dotted lines, respectively. In the saturated state, the dominant mode of the potential is always the zonal mode. On the contrary, the type of the dominant mode of the density depends on the ion temperature. The secondary instabilities of the streamer mode are attributed to the increase of the linear growth rate and are connected to the increase of the ion temperature.

FIG. 7 Plots of the evolution of the energy $\sum_{k} k_{\perp}^{2}\left|\Phi_{k}\right|^{2}$. In the lower panel we see that as the ion temperature increases, the saturation level is increasing as well. This occurs till a certain value of $\tau$. Further increase of the ion temperature leads to a decrease of the saturated level. The decrease in energy during the very initial phase $(t \leq 80)$ is due to the damping of the very short scale modes. Moreover, the "blobs" on the saturation level (upper panel) are signatures of the secondary instabilities of the streamer mode which appear as $\tau$ increases. 
FIGURES

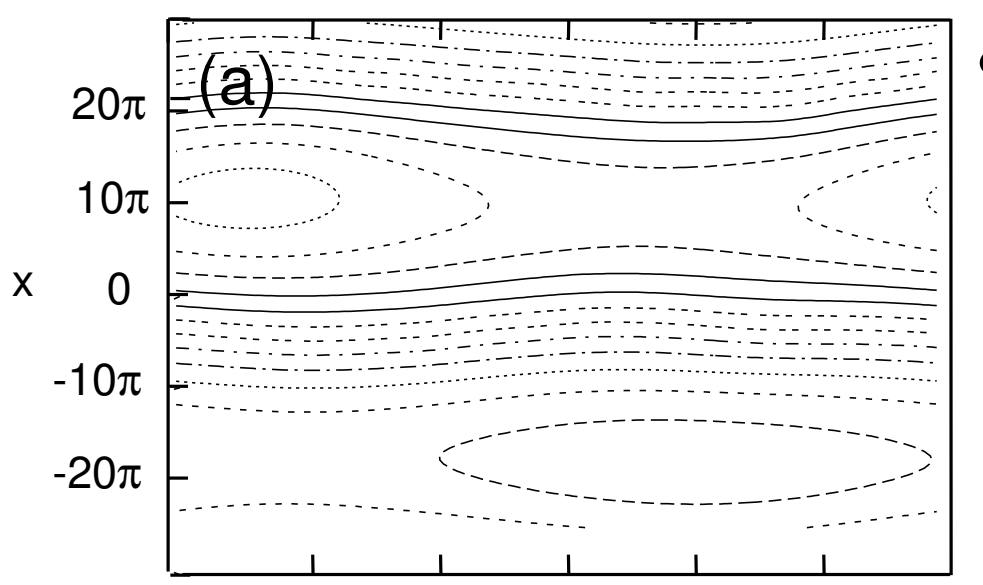

\section{$\Phi(x, y)$}
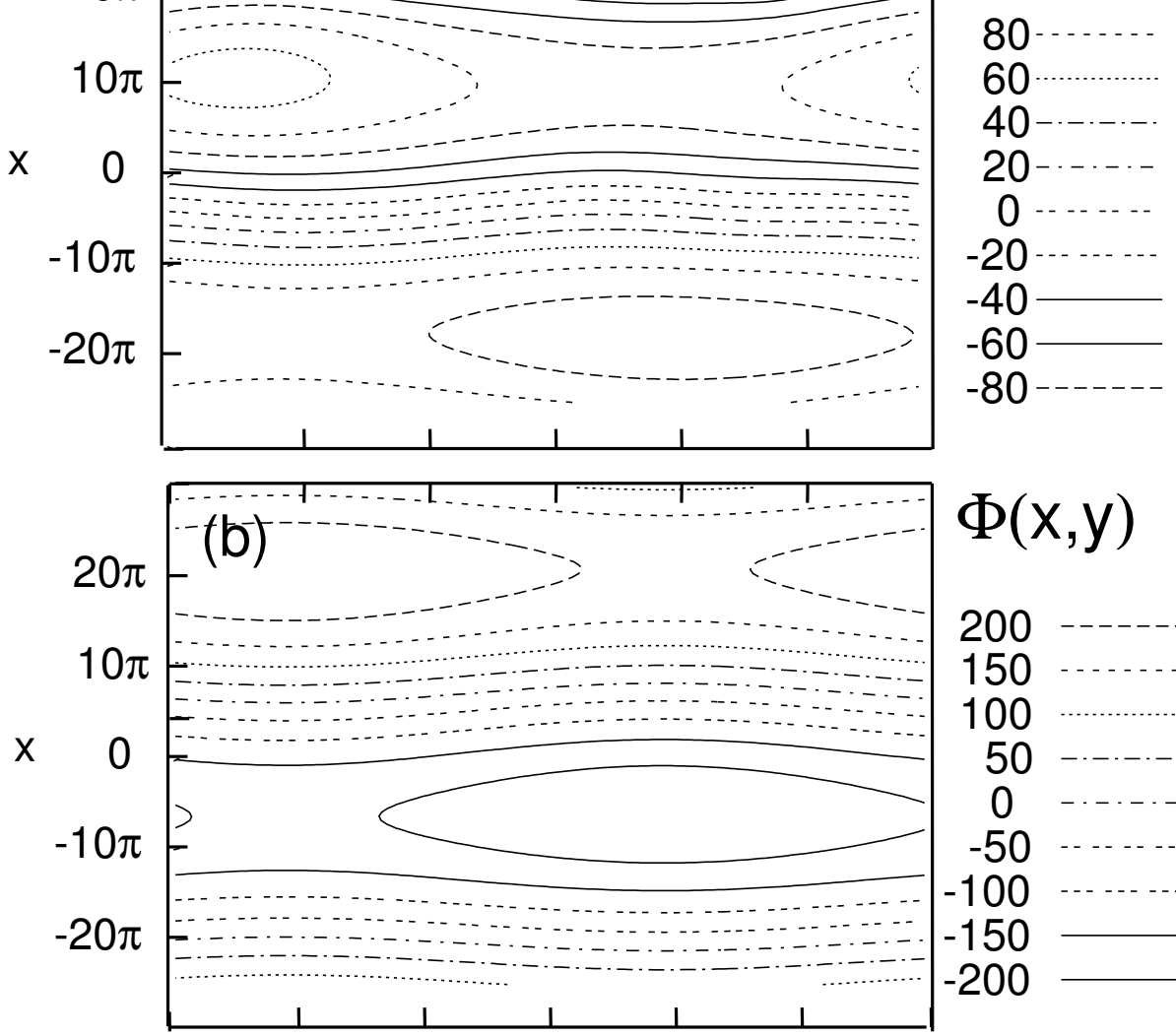

$\Phi(\mathrm{x}, \mathrm{y})$

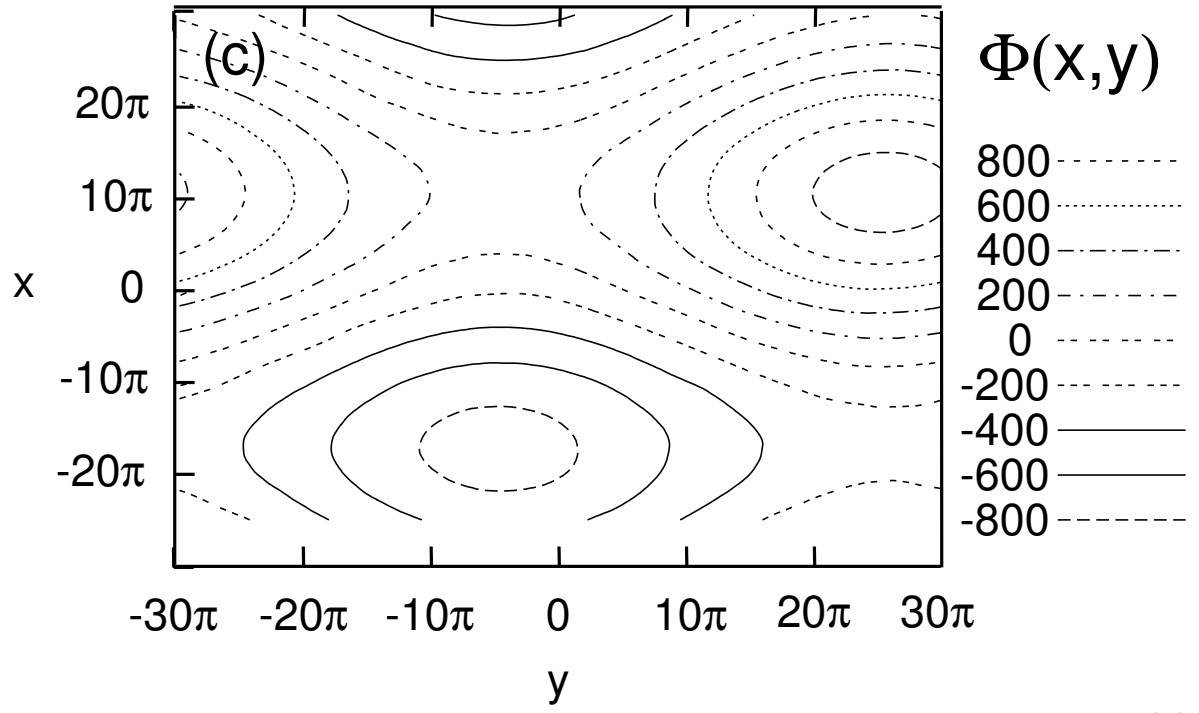

FIG. 1. Iso-contour lines of the potential in the saturation state for (a) $\tau=0$, (b) $\tau=1$, and (c) $\tau=3$. In Subfigs. (a) and (b) the zonal modes largely dominate over the streamer modes. Hence, patterns of zonal flows are formed. For $\tau=3$, the amplitude of the dominant streamer mode is of the same order as the one of the zonal mode, resulting in the pattern of Subfig. (c). 


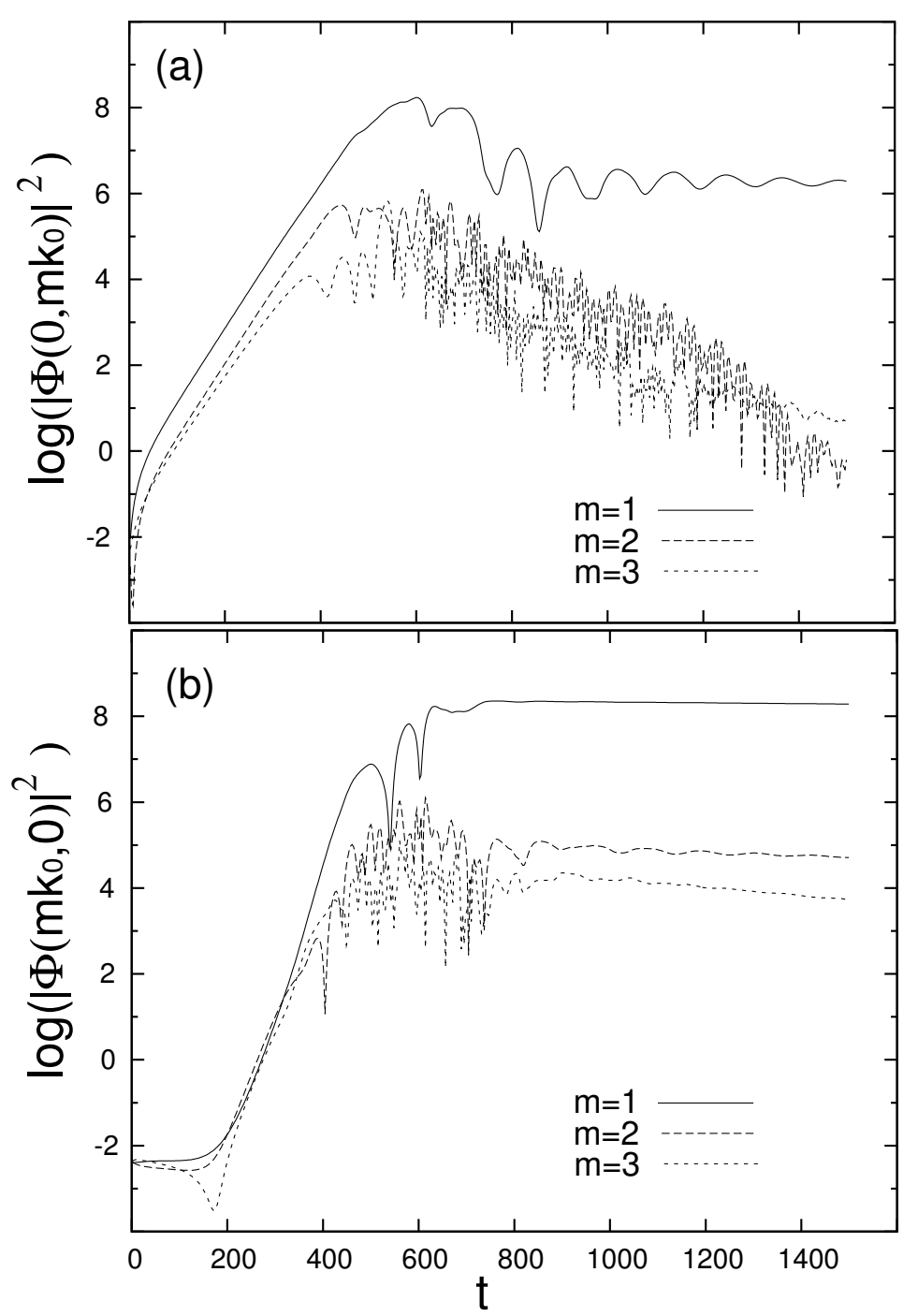

FIG. 2. Evolution and saturation of the largest streamer $\left|\Phi\left(0, m k_{0}\right)\right|^{2}$ (upper panel) and zonal modes $\left|\Phi\left(m k_{0}, 0\right)\right|^{2}$ (lower panel) of the potential. Here, $m=1$ (solid line), $m=2$ (dashed line) and $m=3$ (dotted line) for $\tau=1$, where $k_{0}$ is the minimum wavenumber of the system. The shorter zonal and streamer mode instabilities saturate earlier and at lower amplitudes compared to the largest ones. In the saturated state, the amplitudes of the zonal modes and the largest streamer mode remain constant, while the smaller streamer modes get damped. 


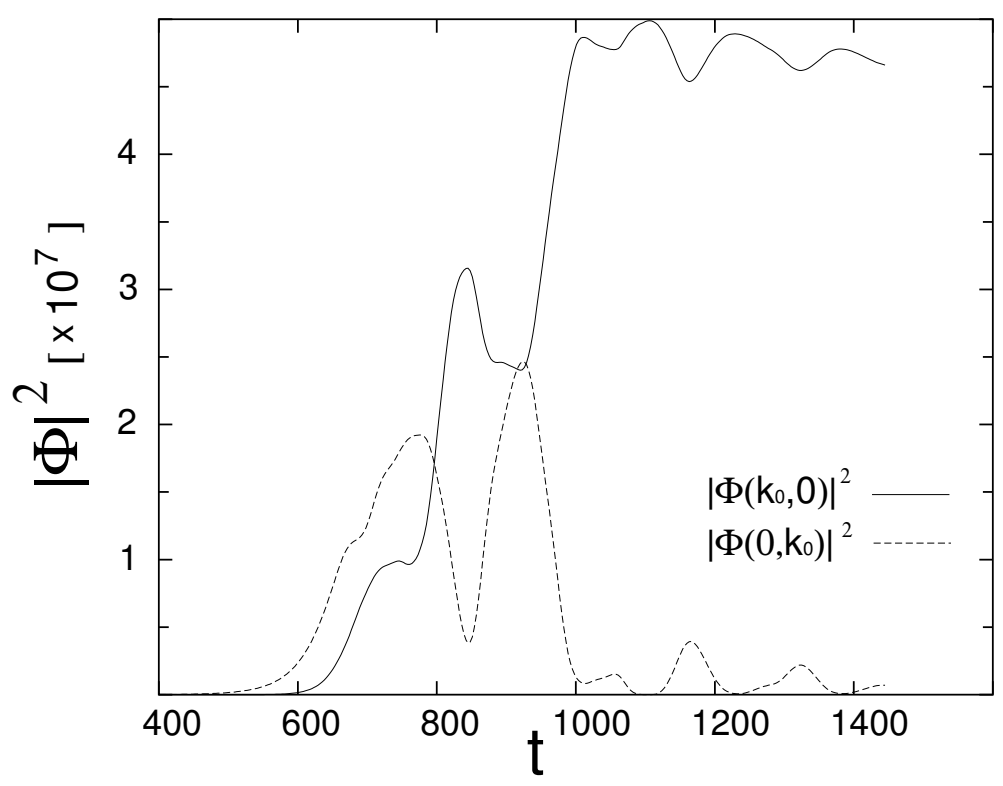

FIG. 3. Oscillating behavior of the dominant anisotropic modes for the potential during the suppression of the instabilities, for $\tau=0$. The zonal mode $\left|\Phi\left(k_{0}, 0\right)\right|^{2}$ is depicted with the solid line, and the streamer mode $\left|\Phi\left(0, k_{0}\right)\right|^{2}$ with the dashed line. 


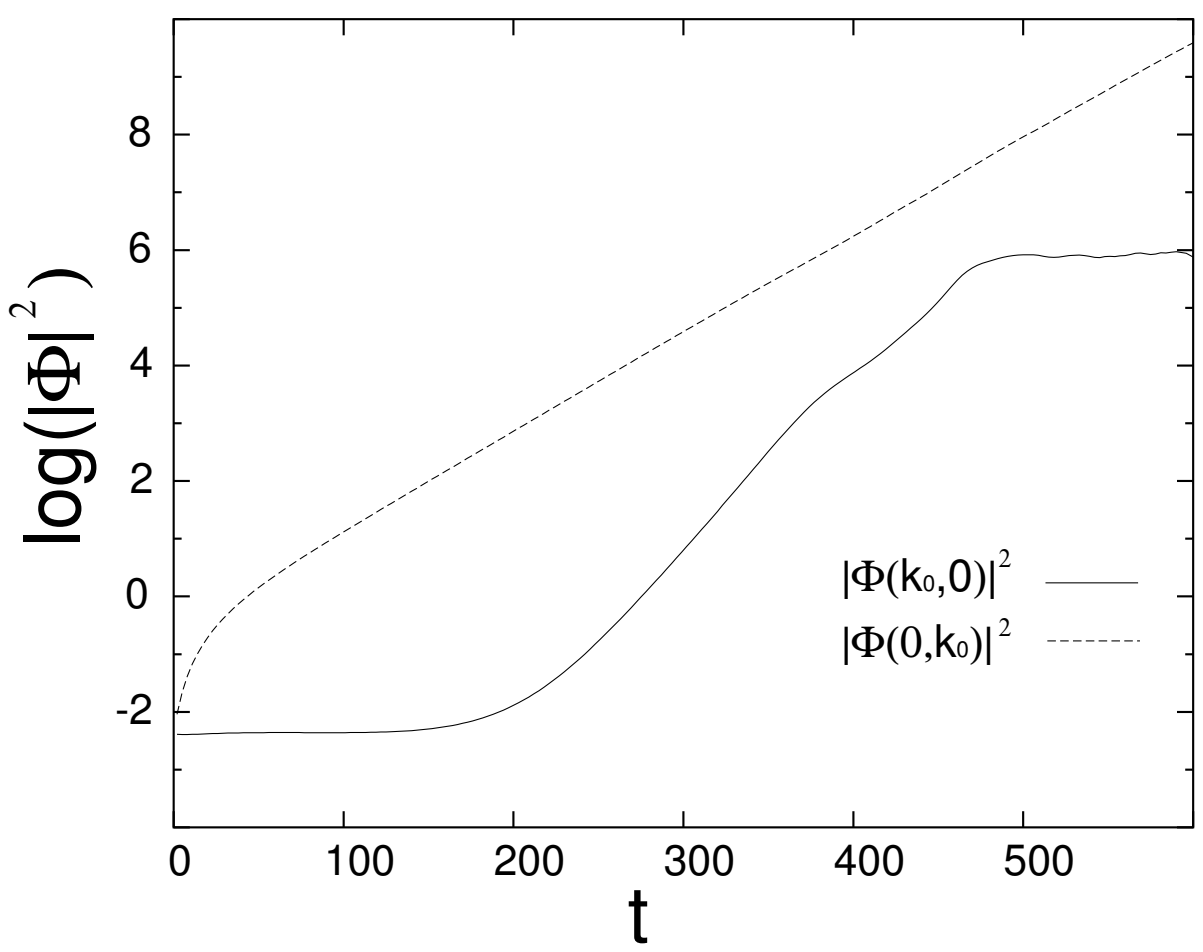

FIG. 4. Evolution of the dominant zonal (solid line) and streamer (dashed line) modes in the absence of the dominant isotropic modes $\vec{k}\left( \pm k_{0}, \pm k_{0}\right)$, for $\tau=1$. The growth of the zonal mode stops at low amplitude, and as a result the dominant flute instability (streamer mode) does not get suppressed. 


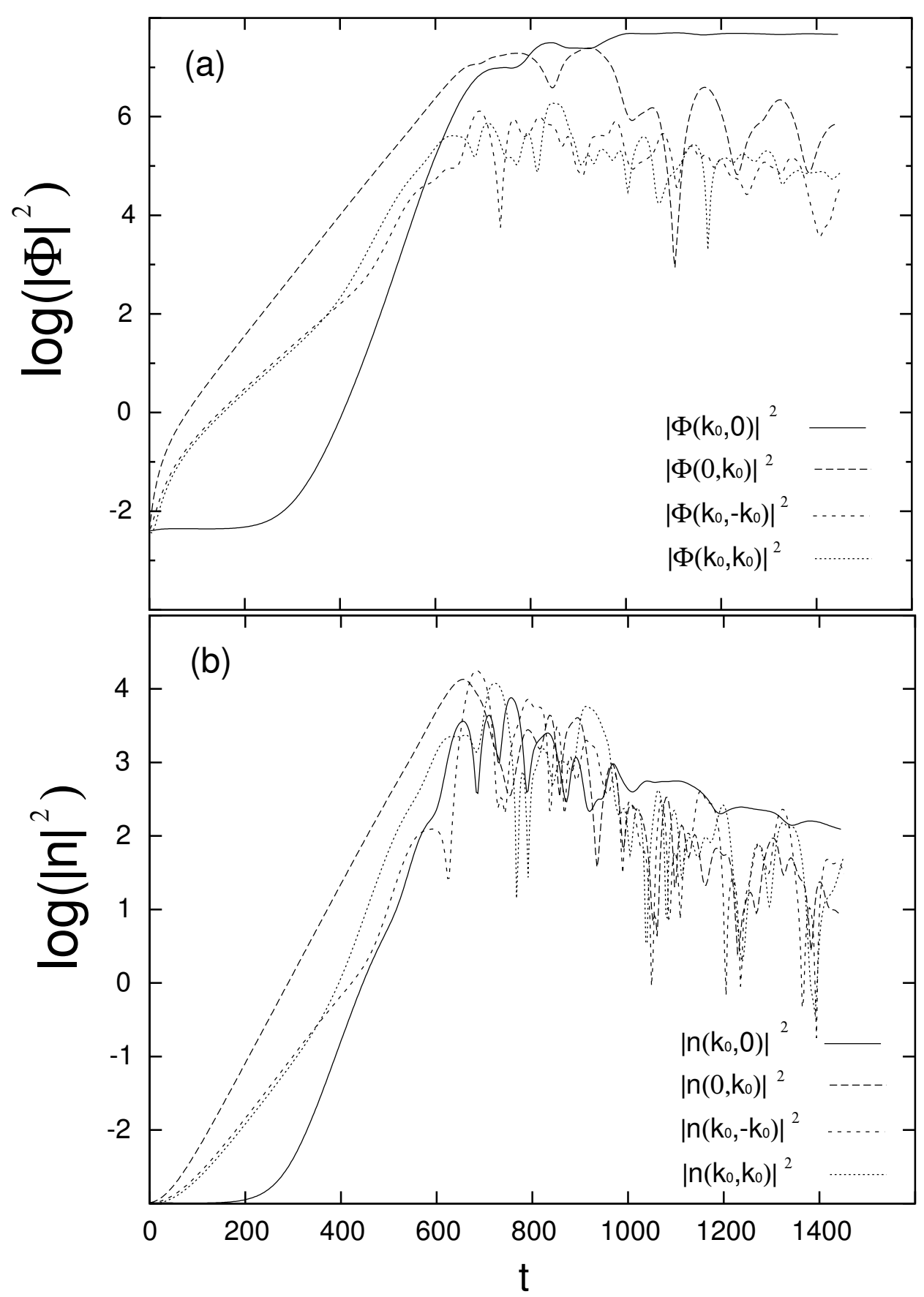

FIG. 5. Temporal evolution and saturation of the dominant isotropic and anisotropic modes for (a) the potential and (b) the density in the cold ion limit. The zonal mode, $\vec{k}\left(k_{0}, 0\right)$ is depicted with solid line, the streamer mode $\vec{k}\left(0, k_{0}\right)$ with dashed line and the isotropic modes $\vec{k}\left(k_{0}, k_{0}\right), \vec{k}\left(k_{0},-k_{0}\right)$ with the smaller dashed and the dotted lines, respectively. The amplitudes of the isotropic modes in the saturation state are significant for the density fluctuations. 

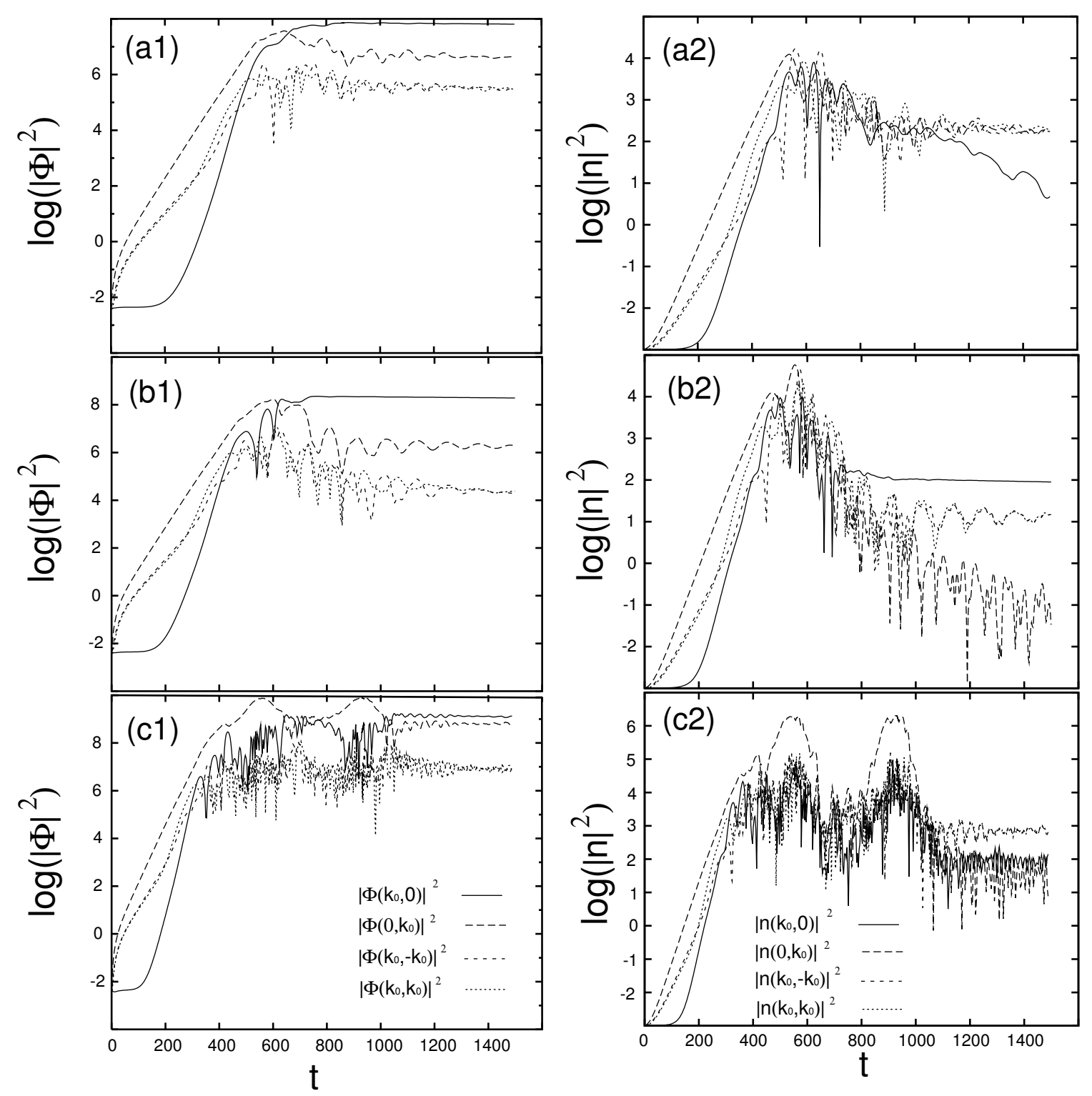

FIG. 6. Evolution of the dominant modes of the potential and the density for different values of the ion temperature: (a) $\tau=0.5$ (b) $\tau=1$ and (c) $\tau=3$. The zonal mode is depicted with solid lines, the streamer mode with dashed lines, and the isotropic modes $\vec{k}\left(k_{0}, k_{0}\right) \vec{k}\left(k_{0},-k_{0}\right)$ with smaller dashed and dotted lines, respectively. In the saturated state, the dominant mode of the potential is always the zonal mode. On the contrary, the type of the dominant mode of the density depends on the ion temperature. The secondary instabilities of the streamer mode are attributed to the increase of the linear growth rate and are connected to the increase of the ion temperature. 

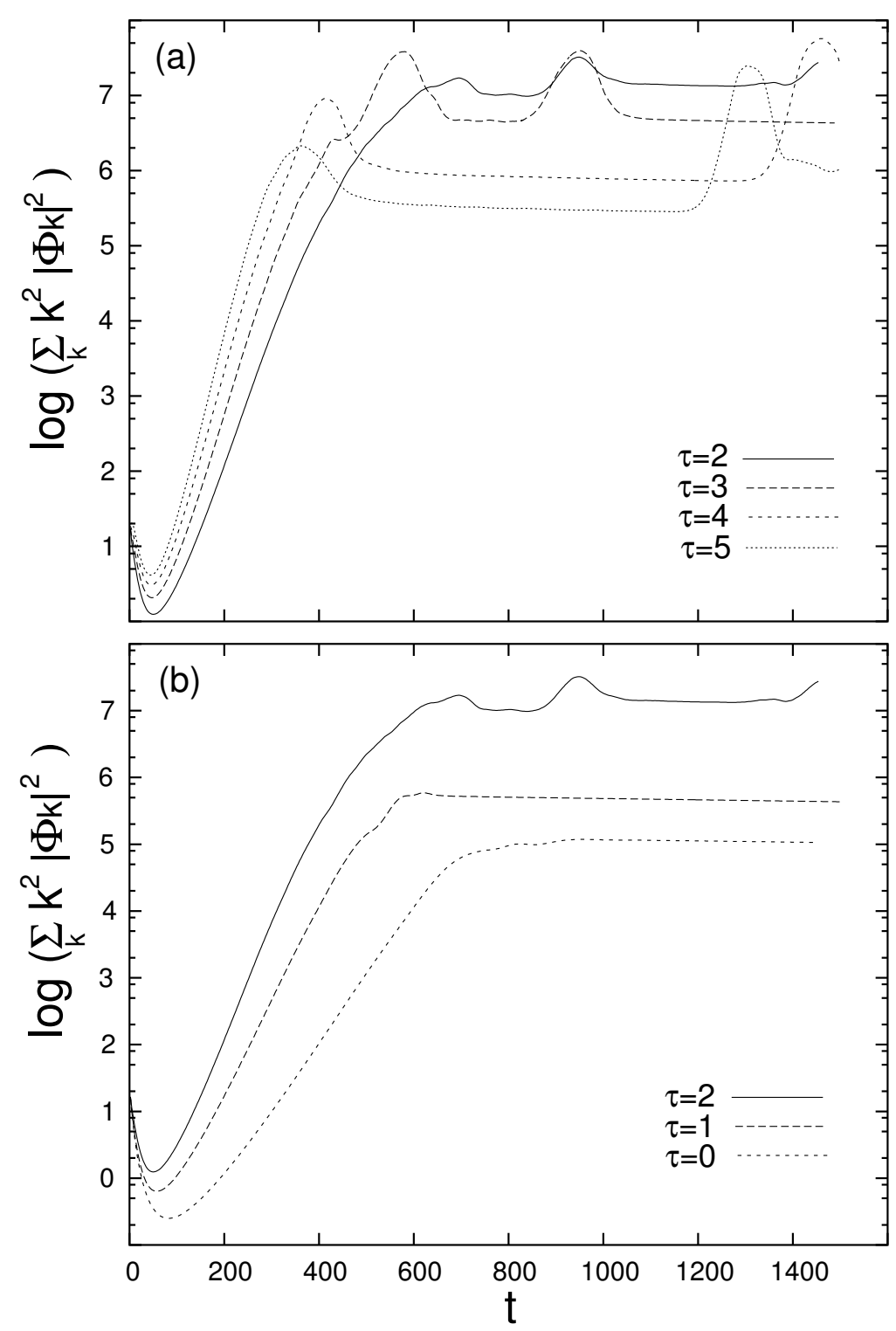

FIG. 7. Plots of the evolution of the energy $\sum_{k} k_{\perp}^{2}\left|\Phi_{k}\right|^{2}$. In the lower panel we see that as the ion temperature increases, the saturation level is increasing as well. This occurs till a certain value of $\tau$. Further increase of the ion temperature leads to a decrease of the saturated level. The decrease in energy during the very initial phase $(t \leq 80)$ is due to the damping of the very short scale modes. Moreover, the "blobs" on the saturation level (upper panel) are signatures of the secondary instabilities of the streamer mode which appear as $\tau$ increases. 